\title{
Pattern formation in a ring cavity with temporally incoherent feedback
}

\author{
Tal Schwartz, Jason W. Fleischer, Oren Cohen, Hrvoje Buljan, and Mordechai Segev \\ Physics Department and Solid State Institute, Technion, Haifa 32000, Israel \\ Tal Carmon \\ California Institute of Technology, Pasadena, California 91125
}

Received January 14, 2004; revised manuscript received May 8, 2004; accepted July 12, 2004

\begin{abstract}
We present a theoretical and experimental study of modulation instability and pattern formation in a passive nonlinear optical cavity that is longer than the coherence length of the light circulating in it. Pattern formation in this cavity exhibits various features of a second-order phase transition, closely resembling laser action. (C) 2004 Optical Society of America

OCIS codes: $190.3100,190.4420$.
\end{abstract}

\section{INTRODUCTION}

Many nonlinear systems in nature exhibit instability of a homogeneous state. For example, in fluid convection systems, chemical reactions, or biological systems, ${ }^{1}$ a homogeneous initial state can break up and transform into a wealth of intricate fine structures that are generally ordered patterns. This is the general trend even though the instability arises from random fluctuations (noise). In many cases the emerging pattern is an array of solitons. $^{2}$ In the past few decades, following the invention of lasers, the phenomenon of spontaneous pattern formation has been observed and extensively studied in various generic optical systems, ${ }^{3,4}$ including active ${ }^{5-9}$ and passive $\mathrm{e}^{10-17}$ nonlinear resonators (cavities) as well as oneway propagation (zero feedback) ${ }^{18-20}$ and counterpropagating schemes. ${ }^{21,22}$ The nonlinearities in such systems can arise from a variety of physical mechanisms: electronic transitions in atomic gas, ${ }^{23}$ semiconductor gain media, ${ }^{9}$ photorefractives ${ }^{10,11,18}$ and thermal ${ }^{16}$ nonlinearities, to name a few. However, in spite of this diversity, the phenomenon of pattern formation is universal, irrespective of the nonlinearities involved, ${ }^{3}$ and occurs also in the temporal domain. ${ }^{13-15}$ In the spatial domain, modulational instability (MI) breaks a uniform beam (a plane wave) into equally spaced filaments that in many cases develop into regular latticelike patterns - typically stripes or hexagons. ${ }^{6-12,16-22}$ As noted above, pattern formation can occur either in cavities or in zero-feedback systems. There are, however, two distinct features distinguishing cavity pattern-formation from patterns evolving during one-way propagation in the absence of any feedback. The first such feature is the existence of a threshold for cavity pattern-formation, below which patterns do not form. This feature is common to all coherent cavities, including passive cavities. ${ }^{3}$ The second feature has to do with the set of resonant frequencies, which is inherent to all coherent cavities. The pattern-formation process is directly affected by the difference between the frequency of the light circulating in the cavity and its nearest resonant frequency (e.g., see Refs. 3 and 6).

Traditionally, pattern formation and MI were investigated in fully coherent cavities, in which the coherence length of the light is much longer than the cavity length. ${ }^{5-17}$ In this case, all the fields circulating in the resonator were not only quasi-monochromatic and spatially coherent but, effectively, also mutually coherent with one another, so the light in the cavity can be treated as a single optical (mean) field..$^{23,24}$ However, recent experimental and theoretical studies ${ }^{25-27}$ have investigated MI and pattern formation in a different kind of cavity: a cavity that is longer than the coherence length of the light circulating in it; that is, the coherence time of the light is much shorter than the time it takes the light to go once around the cavity. Hence fields from different cycles are mutually incoherent, and interference effects between them do not contribute to the nonlinear index change. This means that in such a cavity the circulating fields interact only through their intensities. The main difficulty in analyzing this system results from the fact that the incoherence prohibits the use of the mean-field theory commonly used with coherent cavities. ${ }^{23,25}$ In other words, the recycled fields cannot be represented by a single (averaged) field. Thus a different approach must be taken. ${ }^{25}$ That recent study has shown that patterns do form in such an incoherent cavity, but the pattern-formation process entails several new features that are different from those associated with coherent cavities. $^{25}$ These features, as presented in Ref. 25, are as follows:

1. The modulation depth (visibility) of the pattern experiences a sudden increase above a specific, well-defined threshold. This threshold is different from the threshold of coherent cavities, in the sense that it is not affected by the set of resonant frequencies of the cavity. Therefore the threshold in our cavity is independent of the so-called frequency detuning. ${ }^{3,24}$

2. The emerging pattern exhibits spatial line narrow- 
ing as the feedback or the nonlinearity (or both) are increased, closely resembling the temporal line narrowing in lasers. In lasers, this feature indicates the transition from wide-spectrum fluorescence to lasing (in a narrow temporal bandwidth). ${ }^{28}$

3. The periodicity of the emerging pattern is identical to that of the pattern evolving without feedback under the same parameters. That is, the periodicity of the pattern does not depend on the boundary conditions of the cavity; once again, this feature is in contrast with patterns evolving in coherent cavities. ${ }^{3,11}$

4. The feedback always enhances the visibility of the pattern, even below threshold. This feature, too, is in sharp contrast to passive coherent cavities, ${ }^{3}$ in which patterns are always suppressed below the threshold.

These were the main findings of Ref. 25. The theory presented there was a lowest-order analytic approximation, which accounted for small feedback values and could be employed only below the threshold. Its main findings were corroborated by experiments. Here we analyze the process of MI in a temporally incoherent cavity both below and above the cavity threshold by using a comprehensive approach. We identify several new features and compare these findings with experimental results and with the results of the lowest-order approximation of Ref. 25 .

\section{FORMULATION OF THE PROBLEM}

Let us first describe the optical system under investigation (as illustrated in Fig. 1). A nonlinear medium with noninstantaneous response (e.g., a photorefractive material or a liquid crystal) is placed inside a low-finesse ring cavity in which a spatially coherent, quasimonochromatic light beam is circulating. The beam is made wide enough to cover the entire cross section of the nonlinear medium. The light inside the cavity has a finite coherence length, with a coherence time $\tau_{c}$ that is much shorter than the time required for one round trip in the cavity, both of which are much shorter than the response time of the nonlinearity. The outcome of this hierarchy of characteristic times $\left(\tau_{c} \ll L_{\text {cav }} / c \ll \tau_{\text {material }}\right.$, where $L_{\text {cav }}$ is the cavity length and $c$ is the speed of light) is that in such a cavity, the feedback beams from different cycles are mutually incoherent with one another. However, fields from different cycles, albeit uncorrelated, can interact with one another through the nonlinear element

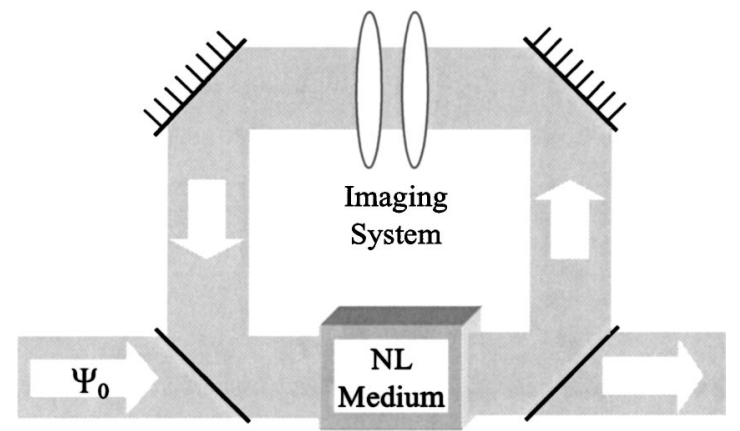

Fig. 1. Schematical sketch of the nonlinear (NL) ring cavity system. in the cavity. The nonlinear medium has an intensitydependent nonlinear index change, $\Delta n$, that responds much slower than the characteristic fluctuation time for the relative phase between beams from different cycles. Thus beams from different cycles interact with one another through $\Delta n=\Delta n(\langle I\rangle)$, where $\langle I\rangle$ is the optical intensity (energy flux) time-averaged over the response time $\tau_{\text {material }}$ of the nonlinearity. Consequently, $\Delta n$ depends on the sum of the intensities of the beams from different cycles but does not depend on interference crossterms between them ${ }^{29}$ (which are averaged out). Thus the resonant frequencies of our cavity, as well as the relative phase between beams from different cycles, do not play any role in the pattern-formation process. Because the cavity has a low finesse ( $\sim 1$ or lower), the cavity cannot act as a filter to narrow the temporal spectrum of the light and increase its coherence length. The feedback loop in our cavity is diffraction free, which is achieved through imaging the output face of the nonlinear medium back to its input face.

The (paraxial) time-independent propagation dynamics of the light inside the nonlinear medium is governed by an infinite set of dimensionless ${ }^{30}$ coupled nonlinear Schrödinger equations:

$$
i \frac{\partial \Psi_{i}}{\partial \zeta}+\frac{\partial^{2} \Psi_{i}}{\partial \xi^{2}}+\Delta n(I) \Psi_{i}=0
$$

where $\Psi_{i}(\xi, \zeta), i=0,1,2, \ldots$, is the envelope of the circulating field from the $i$ th cycle; $\Delta n(I)$ is the nonlinear change in the refractive index, which, as mentioned above, is a function of the time-averaged intensity $I$ $=\left\langle\left|\Sigma_{i=0}^{\infty} \Psi_{i}\right|^{2}\right\rangle=\left\langle\sum_{i, j=0}^{\infty} \Psi_{i}^{*} \Psi_{j}\right\rangle=\sum_{i=0}^{\infty}\left|\Psi_{i}\right|^{2} ; \quad$ and $\zeta$ $=z / z_{0}$ and $\xi=x / x_{0}$ are the normalized propagation and transverse coordinates, respectively. ${ }^{30}$ We emphasize again that interference terms between different recycled fields are averaged out. The boundary conditions imposed by the cavity are $\Psi_{i+1}(\xi, \zeta=0)=\varepsilon \Psi_{i}(\xi, \zeta$ $=L) \exp [i \alpha(t)]$, where $\varepsilon^{2}$ is the intensity feedback in the cavity and $\alpha(t)$ is a randomly fluctuating phase originating from the finite coherence length of the light and varying on a time scale much shorter than the response time of the crystal.

\section{PATTERN FORMATION BELOW THRESHOLD}

First, we investigate analytically the behavior of the system below the threshold and at the transition point. We do this for the self-focusing Kerr-type nonlinearity $[\Delta n(I)=I]$. However, because any other kind of saturable nonlinearity can be linearized around some mean intensity, ${ }^{31}$ we expect to get phenomenologically similar results for other types of nonlinearity as well.

We start by decomposing each field into a uniform beam $\psi$ and a spatially dependent perturbation $\phi(\xi, \zeta)$ on it (assuming that the perturbation amplitude is much smaller than the amplitude of the homogenous background) and take their mutual propagation as $\Psi_{i}=\left[\psi_{i}\right.$ $\left.+\phi_{i}(\xi, \zeta)\right] \exp \left(i \Gamma_{i} \zeta\right)$. We expand the total intensity in the limit $\left|\phi_{i}\right| \ll\left|\psi_{i}\right|$ : 


$$
\begin{aligned}
I= & \sum_{i=0}^{\infty}\left|\psi_{i}+\phi_{i}\right|^{2}=\sum_{i}\left[\left|\psi_{i}\right|^{2}+\psi_{i}^{*} \phi_{i}+\psi_{i} \phi_{i}^{*}\right. \\
& \left.+O\left(\phi_{i}{ }^{2}\right)\right] \cong \frac{\left|\psi_{0}\right|^{2}}{1-\varepsilon^{2}}+\sum_{i}\left(\psi_{i}^{*} \phi_{i}+\psi_{i} \phi_{i}^{*}\right),
\end{aligned}
$$

where $\left|\psi_{0}\right|^{2}$ is the intensity of the beam entering the cavity, and we have employed the boundary conditions $\left|\psi_{i+1}\right|^{2}=\varepsilon^{2}\left|\psi_{i}\right|^{2}=\varepsilon^{2(i+1)}\left|\psi_{0}\right|^{2}$ or $\left|\psi_{i}\right|^{2}=\varepsilon^{2 i}\left|\psi_{0}\right|^{2}$ for the intensity. After substituting the fields and the nonlinearity into Eq. (1), we get

$$
\begin{aligned}
& -\Gamma_{i}\left(\psi_{i}+\phi_{i}\right)+i \frac{\partial \phi_{i}}{\partial \zeta}+\frac{\partial^{2} \phi_{i}}{\partial \xi^{2}} \\
& +\left[\frac{\left|\psi_{0}\right|^{2}}{1-\varepsilon^{2}}+\sum_{j}\left(\psi_{j}^{*} \phi_{j}+\psi_{j} \phi_{j}^{*}\right)\right]\left(\psi_{i}+\phi_{i}\right)=0 .
\end{aligned}
$$

By collecting leading-order terms only, we have

$$
-\Gamma_{i} \psi_{i}+\frac{\left|\psi_{0}\right|^{2}}{1-\varepsilon^{2}} \psi_{i}=0
$$

from which we find that all the propagation constants are identical with $\Gamma_{i}=\left|\psi_{0}\right|^{2} /\left(1-\varepsilon^{2}\right)$. Substituting this back into Eq. (3) and taking the first-order terms, we finally get an infinite set of coupled linearized equations governing the evolution of the small perturbations in the cavity:

$$
i \frac{\partial \phi_{i}}{\partial \zeta}+\frac{\partial^{2} \phi_{i}}{\partial \xi^{2}}+\sum_{j}\left(\psi_{j}^{*} \phi_{j}+\psi_{j} \phi_{j}^{*}\right) \psi_{i}=0 .
$$

Normalizing each perturbation by its jointly propagating uniform fields $\bar{\phi}_{i}=\phi_{i} / \psi_{i}$ gives

$$
i \frac{\partial \bar{\phi}_{i}}{\partial \zeta}+\frac{\partial^{2} \bar{\phi}_{i}}{\partial \xi^{2}}+\sum_{j}\left|\psi_{j}\right|^{2}\left(\bar{\phi}_{j}+\bar{\phi}_{j}^{*}\right)=0 .
$$

It is important to note that this set of equations is invariant under an arbitrary change of phase of any of the circulating fields, that is, $\Psi_{i}=\psi_{i}\left(1+\bar{\phi}_{i}\right) \rightarrow \Psi_{i} \exp (i \theta)$, owing to the fact that interference terms are averaged out to zero. As a result of this invariance, we can choose all $\psi_{i}$, without the loss of generality, to be real and to omit the absolute value sign on $\psi_{i}$. Using this, we can now write the boundary conditions for the fields (rather than for the intensity) as $\bar{\phi}_{i+1}(\xi, \zeta=0)=\bar{\phi}_{i}(\xi, L)$ and $\psi_{i}=\varepsilon^{i} \psi_{0}$. This simplification is a result of the direct imaging (output face to input face) in the feedback loop, which maintains the relative phase between the dc component and the perturbation within the same cycle. In other words, the relative phase between $\psi_{i}$ and $\phi_{i}$ is deterministic, although fields from different cycles $\{i, j\}$ are mutually incoherent and their relative phase is stochastic. Hence we may rewrite Eq. (6) in the form

$$
i \frac{\partial \bar{\phi}_{i}}{\partial \zeta}+\frac{\partial^{2} \bar{\phi}_{i}}{\partial \xi^{2}}+\psi_{0}^{2} \sum_{j} \varepsilon^{2 j}\left(\bar{\phi}_{j}+\bar{\phi}_{j}^{*}\right)=0 .
$$

Next, we separate the real and imaginary parts of the perturbations by defining $\bar{\phi}_{i}{ }^{+} \equiv \bar{\phi}_{i}+\bar{\phi}_{i}{ }^{*}, \bar{\phi}_{i}{ }^{-} \equiv i\left(\bar{\phi}_{i}\right.$ $\left.-\bar{\phi}_{i}{ }^{*}\right)$. Adding and subtracting Eq. (7) and its complex conjugate yields two coupled sets of equations:

$$
\begin{array}{r}
\frac{\partial \overline{\boldsymbol{\phi}}_{i}^{-}}{\partial \zeta}+\frac{\partial^{2} \bar{\phi}_{i}^{+}}{\partial \xi^{2}}+2 \psi_{0}^{2} \sum_{j} \varepsilon^{2 j} \bar{\phi}_{j}^{+}=0, \\
\frac{\partial \bar{\phi}_{i}^{+}}{\partial \zeta}-\frac{\partial^{2} \bar{\phi}_{i}^{-}}{\partial \xi^{2}}=0 .
\end{array}
$$

We now define two new, global functions $\Phi(\xi, \zeta)$ $\equiv \psi_{0}^{2} \Sigma_{i} \varepsilon^{2 i} \bar{\phi}_{i}^{+}$and $\Theta(\xi, \zeta) \equiv \psi_{0}{ }^{2} \Sigma_{i} \varepsilon^{2 i} \bar{\phi}_{i}{ }^{-}$. Multiplying each one of the sets in Eqs. (8) by $\psi_{0}^{2} \varepsilon^{2 i}$ and summing over all $i$ 's reduce Eqs. (8) to only two equations, describing the coupled evolution of $\Phi$ and $\Theta$ :

$$
\begin{aligned}
\frac{\partial \Theta}{\partial \zeta}+\frac{\partial^{2} \Phi}{\partial \xi^{2}}+\frac{2 \psi_{0}{ }^{2}}{1-\varepsilon^{2}} \Phi & =0, \\
\frac{\partial \Phi}{\partial \zeta}-\frac{\partial^{2} \Theta}{\partial \xi^{2}} & =0 .
\end{aligned}
$$

We note that, with the definition of $\bar{\phi}_{i}{ }^{+}$, the total intensity inside the cavity can be written as

$$
\begin{aligned}
I & =\frac{\psi_{0}^{2}}{1-\varepsilon^{2}}+\sum_{i} \psi_{i}{ }^{2}\left(\bar{\phi}_{i}+\bar{\phi}_{i}{ }^{*}\right) \\
& =\frac{\psi_{0}^{2}}{1-\varepsilon^{2}}+\psi_{0}^{2} \sum_{i} \varepsilon^{2 i} \bar{\phi}_{i}^{+} \\
& =\frac{\psi_{0}^{2}}{1-\varepsilon^{2}}+\Phi(\xi, \zeta),
\end{aligned}
$$

meaning that $\Phi(\xi, \zeta)$ is just the total perturbation of the intensity, which is the sum of all contributions from all the recycled fields, added incoherently. In other words, rather than representing the electric fields circulating in the cavity by a single mean electric field, we present a global intensity field accounting for all cycles. In order to decouple Eqs. (9), we first transform to Fourier space along the transverse coordinate $\left(\partial^{2} / \partial \xi^{2} \rightarrow-q^{2}\right)$

$$
\begin{aligned}
\frac{\partial \hat{\Theta}}{\partial \zeta}-q^{2} \hat{\Phi}+\frac{2 \psi_{0}^{2}}{1-\varepsilon^{2}} \hat{\Phi}=0 & \\
\frac{\partial \hat{\Phi}}{\partial \zeta}+q^{2} \hat{\Theta} & =0
\end{aligned}
$$

where $\hat{\Phi}(q, \zeta)=\int_{-\infty}^{\infty} \Phi(\xi, \zeta) \exp (-i q \xi) \mathrm{d} \xi$ and $\hat{\Theta}(q, \zeta)$ $=\int_{-\infty}^{\infty} \Theta(\xi, \zeta) \exp (-i q \xi) \mathrm{d} \xi$ are the Fourier transforms of $\Phi$ and $\Theta$, respectively, and $q$ is the perturbation wave number (i.e., the momentum in the transverse plane). We then take the second derivative of $\hat{\Phi}$ with respect to $\zeta$, which is, according to Eqs. (11), $\partial^{2} \hat{\Phi} / \partial \zeta^{2}=-q^{2}(\partial \hat{\Theta} / \partial \zeta)$ or

$$
\frac{\partial^{2} \hat{\Phi}}{\partial \zeta^{2}}=q^{2}\left(\frac{2 \psi_{0}^{2}}{1-\varepsilon^{2}}-q^{2}\right) \hat{\Phi} \equiv g^{2} \hat{\Phi}
$$

where $g^{2}(q)=q^{2}\left[2 \psi_{0}^{2} /\left(1-\varepsilon^{2}\right)-q^{2}\right]$ is real. Equation (12) has exponentially growing/decaying or oscillatory solutions, depending on the sign of $g^{2}(q)$. To observe some pattern at the output face of the nonlinear medium, we 

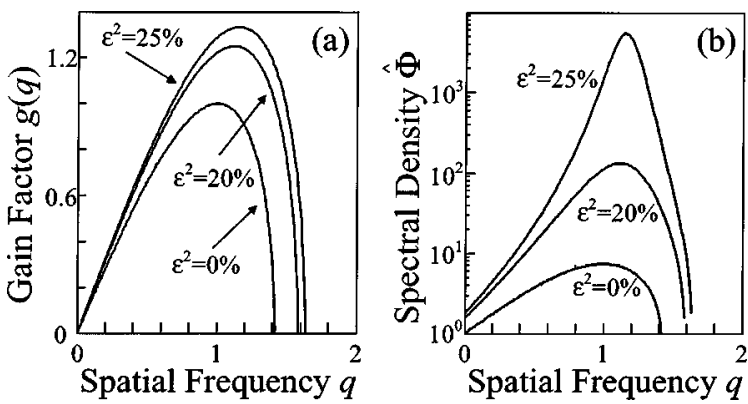

Fig. 2. (a) Calculated exponential gain factor $g(q)$ and (b) spatial spectral density of the perturbation at the output face of the nonlinear medium for different intensity feedback values below threshold, based on the analytic solution (calculated with $\psi_{0}$ $=1$ and $L=1$ )

require that $g(q)$ (denoting the gain of each spatial frequency) be real and positive; hence small perturbations at the input will be amplified along propagation. Assuming such growing perturbations, that is, those with real $g(q)$ values, the general solutions for the global fields are

$$
\begin{aligned}
& \hat{\Phi}(q, \zeta)=A \exp (g \zeta)+B \exp (-g \zeta), \\
& \hat{\Theta}(q, \zeta)=\frac{i g}{q^{2}}[A \exp (g \zeta)-B \exp (-g \zeta)] .
\end{aligned}
$$

It can be shown that the boundary conditions translate into the global fields as $\hat{\Phi}(q, \zeta=0)=\varepsilon^{2} \hat{\Phi}(q, \zeta=L)$ $+\delta$ and $\hat{\Theta}(q, \zeta=0)=\varepsilon^{2} \hat{\Theta}(q, \zeta=L)+\gamma$, where $\delta(\gamma)$ is the real (imaginary) part of the perturbation on the incoming beam. With these boundary conditions, we can solve for the coefficients in Eqs. (13). We find that the solution for the exponentially growing part of the perturbation, at the output face of the medium, is

$$
\begin{aligned}
\hat{\Phi}(q, L) & \propto \frac{\exp [g(q) L]}{1-\varepsilon^{2} \exp [g(q) L]}, \\
g(q) & =|q|\left(\frac{2 \psi_{0}^{2}}{1-\varepsilon^{2}}-q^{2}\right)^{1 / 2} .
\end{aligned}
$$

This form is identical to the solution presented in Ref. 25; however, the exponential gain factor has been corrected, as the intensity of the incoming beam is now multiplied by $1 /\left(1-\varepsilon^{2}\right)$, which is identified with the sum of intensities of all the cycles in the cavity. Notice that all the above expressions depend on the intensity feedback $\varepsilon^{2}$ and not on the field feedback directly, and that it is insensitive to the feedback phase delay. This results from the fact that the cavity is incoherent and interference terms do not contribute to the interplay between different cycles.

Examining expression (14), which is the spectral density of the outgoing perturbation, reveals several facts. These are illustrated in Figs. 2 and 3 (calculated with the parameters $\psi_{0}=1$ and $L=1$ ):

1. Figure 2 shows the exponential gain factor, $g(q)$, and the spectral density of the perturbations at the output face of the medium, $\hat{\Phi}(q, L)$ (on a logarithmic scale), for different values of the intensity feedback. When the feedback is zero (no cavity), there are exponentially growing perturbations with their spectral densities centered around $q_{\max }=\psi_{0}$. However, as one starts to increase the feedback, the spatial frequency with the highest exponential gain factor is shifted according to $q_{\max }$ $=\psi_{0} / \sqrt{1-\varepsilon^{2}}$. Hence the dominant spatial frequency in the cavity [which is identical to the one with the highest gain factor $g(q)$ ] does depend on the boundary conditions, in contrast to the result of the lowest-order (small feedback) theory. ${ }^{25}$ It is important to note that the main effect of creating a cavity (by closing a feedback loop) is the dependence of the total amplification of the spectral density on the feedback in the cavity. The other effect, namely, the increased nonlinearity due to increased intensity (resulting from the feedback) is relatively minor. That is, the amplification originates mainly from recycling the perturbation in the cavity and the interplay between different cycles.

2. At some definite threshold value of the cavity feedback, which we denote as $\varepsilon_{\text {th }}$, the amplitude of the perturbation at the wave number with the highest gain factor (solid curve in Fig. 3) diverges. This threshold occurs at the point at which the exponential gain induced by the nonlinearity $\exp (g L)$ exactly balances the intensity losses in the cavity (which are quantified here by a loss parameter $1 / \varepsilon^{2}$ ). At this critical feedback value and above it, the analytic linear stability analysis presented here is no longer valid, and, as will be shown later, a periodic pattern with modulation depth independent of the initial noise is formed, in contrast to the situation below the threshold.

3. The spectral width of the perturbation ${ }^{32}$ (dashed curve in Fig. 3) decreases monotonically, meaning that the intensity pattern is becoming more ordered and closer to a perfectly periodic structure as the feedback is increased. Also, the amplitude of the perturbation (or the modulation depth) increases monotonically as the feedback is increased.

It is interesting to note that, after our solving for $A$ and $B$ in Eqs. (13), the solution for the total intensity perturbation $\hat{\Phi}(\zeta)$ can be used to obtain a separate equation for each $\bar{\phi}_{i}{ }^{+}$:

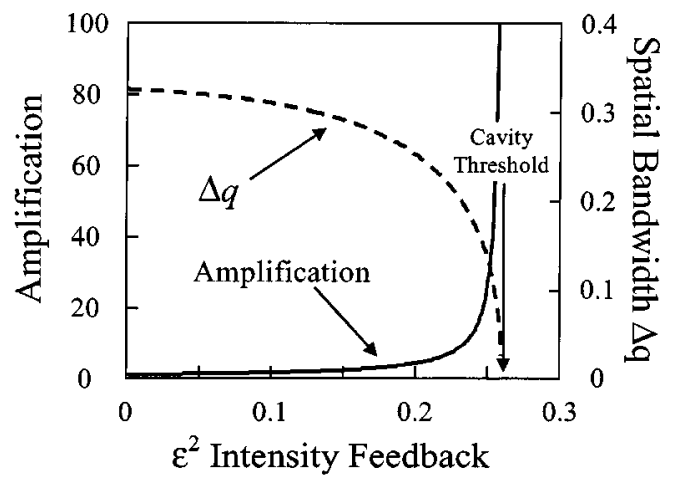

Fig. 3. Amplitude of the dominant spatial frequency at the output of the nonlinear medium (solid curve) and the line narrowing of the spatial bandwidth $\Delta q$ (dashed curve) as a function of the feedback in the cavity below the threshold, based on the analytic solution. The cavity threshold is marked by an arrow (calculated with $\psi_{0}=1$ and $L=1$ ). 


$$
\frac{\partial^{2} \hat{\phi}_{i}^{+}}{\partial \zeta^{2}}+q^{4} \hat{\phi}_{i}^{+}=2 q^{2}[A \exp (g \zeta)+B \exp (-g \zeta)]
$$

This is an inhomogeneous ordinary deferential equation with an exponential particular solution proportional to $A$ and $B$, which indicates that all the cycles are diverging collectively. In addition, it also has an oscillatory solution, indicating that part of the perturbation in each separate field is coupled into an oscillating mode $\exp \left( \pm i q^{2} \zeta\right)$. However, as was shown above, these (phase-dependent) oscillations cancel out when summed over all cycles and do not contribute to the intensity pattern evolving in the cavity.

\section{PATTERN FORMATION ABOVE THRESHOLD}

In order to reveal and understand the behavior of the system above the threshold, where the above expressions do not hold, we simulate the propagation of light inside the cavity under the full nonlinear equations [Eq. (1)] by using a standard beam propagation method (BPM). We use the method iteratively as follows: We start with the incoming beam $\Psi_{0}(\xi, \zeta=0)$, which is held at the input as a uniform beam (whose intensity we denote by $I_{0}$ $\equiv \psi_{0}^{2}$ ). We introduce weak white noise (of a random amplitude and phase) on top of $\psi_{0}$ and calculate its nonlinear propagation for a distance $\zeta=L$ (output face). We then add the second cycle by using $\Psi_{1}(\xi, \zeta=0)$ $=\varepsilon \Psi_{0}(\xi, \zeta=L)$ and calculate their coupled propagation through the nonlinear medium. Then $\Psi_{1}(\xi, \zeta=0)$ is corrected, by use of the new solution for $\Psi_{0}(\xi, \zeta$ $=L$ ), a third cycle is added, and so forth; we add another field at each iteration and update the input of the previous fields by using the boundary conditions $\Psi_{i+1}(\xi, \zeta$ $=0)=\varepsilon \Psi_{i}(\xi, \zeta=L)$. We add up fields up to feedback value of $\varepsilon^{i}<10^{-6}$, where $i$ is the cycle number, and continue the iterative collective propagation of these cycles until the set of $\left\{\Psi_{i}(\xi, \zeta)\right\}$ converges [we arbitrarily define the code convergence when the relative change in the (output face) power spectrum in the particular iteration is smaller than $0.01 \%$ ].

The incoherence of the light is manifested in the omission of interference terms from the expression describing the intensity. In the transverse dimension we use 2048 sampling points over a (dimensionless) width of 400 , meaning that the resolution in $k$ space is $\Delta k=0.015$ and the maximal spatial frequency is $k_{\max }=16.08$.

First, we use the Kerr-type nonlinearity $[\Delta n(I)=I]$. We set $I_{0}=1$ for the mean intensity of the incoming beam (which sets the strength of the nonlinearity) and $L=2$ for the nonlinear propagation distance. Figure 4 shows the total intensity distribution (normalized by the mean intensity in the cavity) at the output face of the medium. When there is no feedback, the intensity remains almost uniform, and the fluctuations are weak. When we set the intensity feedback to $10 \%$, slightly below threshold, the instability is more pronounced, and a quasiperiodic low-visibility intensity modulation emerges. Increasing the feedback value to $\varepsilon^{2}=11 \%$, slightly above threshold, results in a sudden increase of the modulation depth of the intensity pattern, which assumes a more pro-

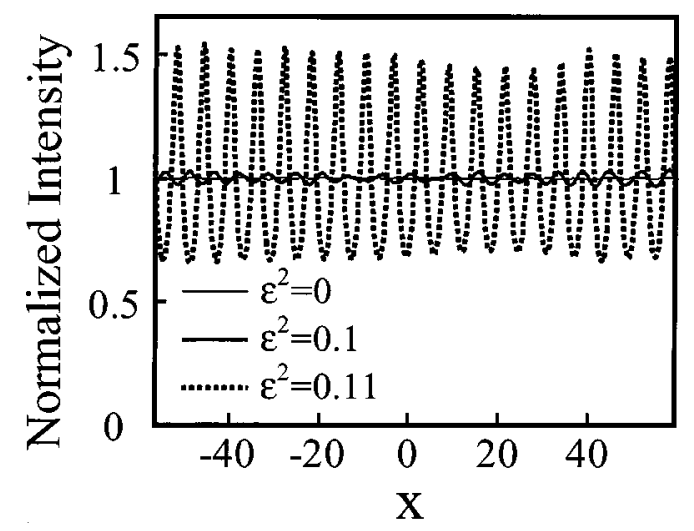

Fig. 4. Typical results of the numerical simulation with Kerr nonlinearity, showing the intensity pattern at the output of the nonlinear medium (normalized by the mean intensity in the cavity) without feedback (thin curve), slightly below the cavity threshold (thick curve), and slightly above the threshold (dashed curve), demonstrating the sudden increase in the modulation depth.

nounced periodicity and becomes highly regular. This sudden change clearly indicates that a cavity threshold had been passed and the instability went through a transition in its nature. Notice that the analytically calculated value of the threshold intensity feedback [found by equating the denominator of expression (14) to zero with the above parameters] is $10.67 \%$, in accordance with the results of the numerical simulation.

In order to study and quantify the behavior of the cavity as the feedback is varied, we perform the numerical simulations with small increments of the cavity feedback under the same parameters (length and intensity) as in Fig. 4. We perform the simulations with three strengths of initial noise $(0.2 \%, 1 \%$, and $5 \%$ of the mean intensity). As an order parameter, we choose $\sqrt{2} \sigma_{I} /\langle I\rangle$, where $\sigma_{I}$ $=\left\{\int[I(x)-\langle I\rangle]^{2} \mathrm{~d} x\right\}^{1 / 2}$ is the standard deviation of the intensity and $\langle I\rangle$ is the average intensity, as a measure for the calculated visibility of the emerging pattern. This parameter is related to the spatial correlation function and is more suitable for statistical calculations. It reduces to the usual expression for the visibility $\left[\left(I_{\max }\right.\right.$ $\left.\left.-I_{\min }\right) /\left(I_{\max }+I_{\min }\right)\right]$ when the intensity pattern is perfectly sinusoidal. The results are presented in Fig. 5. Generally, below the cavity threshold (also shown magnified in the inset), the perturbations are weakly amplified, and their (small) amplitude is proportional to the initial noise strength. As the cavity threshold is approached, perturbations with a particular spatial frequency start diverging, as predicted by the theoretical model. However, above threshold, the divergence of the perturbations is arrested by the nonlinearity, resulting in periodic perturbations with finite amplitude depending only on the feedback, and completely independent of the noise entering the cavity. This is a typical behavior of a second-order phase transition (e.g., laser action ${ }^{33}$ )—as the control parameter (feedback in our case, pump intensity or temperature in other examples) is varied, the fluctuations of the disordered system diverge toward a critical value of the control parameter. As this critical value is passed, the system spontaneously becomes organized, and the order parameter relaxes to some finite value that is inher- 
ent to the system and depends on the system's parameters alone.

Another characteristic of the state of the system is the bandwidth of the spatial power spectrum of the emerging perturbation. Figure 6 shows the spectral width ${ }^{32}$ as a function of the intensity feedback $\varepsilon^{2}$. Without feedback, the width of the spatial spectrum is relatively high $(\sim 0.4)$ and corresponds to the width of the natural gain $[\exp (g L)]$ resulting from the nonlinearity. When the feedback is increased, the width of the spectrum decreases monotonically toward the cavity threshold. The dependence of the bandwidth on feedback in this region, predicted by the analytical model presented above, can be calculated with expression (14) (solid curve in Fig. 6). This indicates that the incoherent cavity is well described by our analytical model. Above the threshold (which is designated by an inflection point of the curve), there is a further narrowing of the bandwidth until some minimal width is approached. This again indicates a behavior similar to that of a laser, with the transverse direction replacing the longitudinal (or the temporal) direction. In lasers, line narrowing occurs at the transition from spontaneous emission (wide temporal spectrum) to lasing (narrowband with a minimal width known as the Schawlow-Townes limit ${ }^{28}$ ), as the pump is increased. In

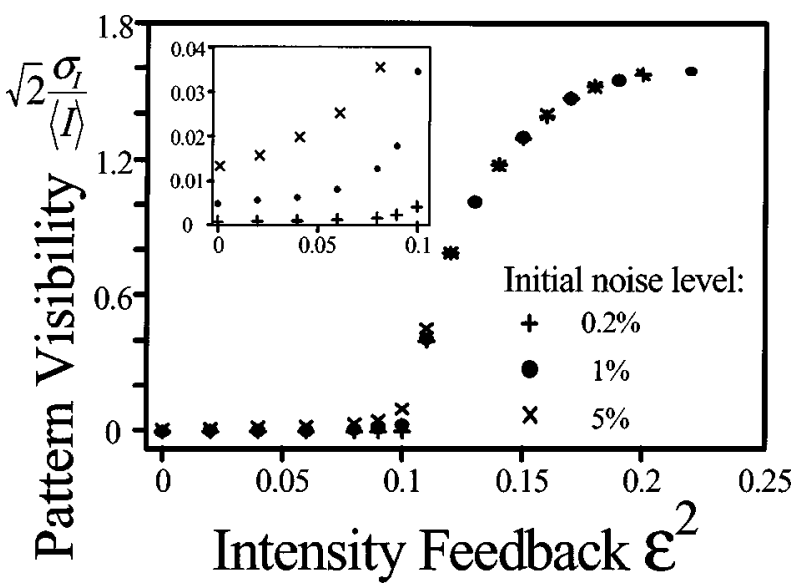

Fig. 5. Calculated visibility versus intensity feedback for the numerical simulations with the Kerr nonlinearity, with different strengths of initial noise, showing the transition from lowvisibility perturbations (below threshold) to a highly modulated intensity pattern (above threshold). The inset shows the magnification of the region below the cavity threshold.

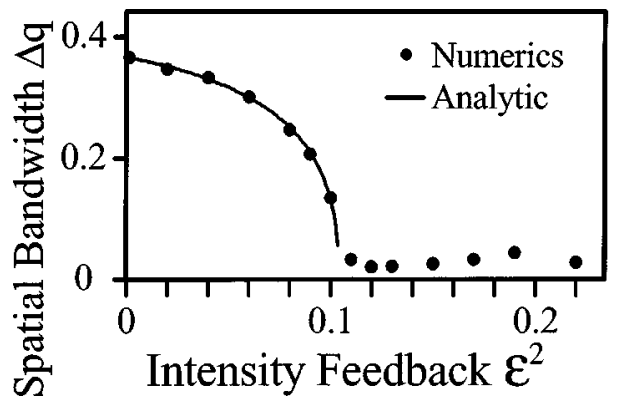

Fig. 6. Bandwidth of the perturbations' spatial spectrum versus intensity feedback calculated with the numerical simulation (circles) below and above the threshold, and with the analytic solution (solid curve) below the cavity threshold for Kerr nonlinearity, showing line narrowing as the feedback is increased. our case, the transition is between the wideband amplification of spatial perturbations and the emergence of a spatially periodic pattern with a definite periodicity.

Further increase of the feedback brings up high-order nonlinear effects, generating higher harmonics and leading, by their interplay, to some broadening of the spectrum. At values of the intensity feedback that are larger than $\sim 25 \%$, our numerical code does not converge. This could possibly indicate a secondary instability. However, to determine whether the instability is indeed physical or merely a numerical artifact, further studies are required, with computational power exceeding our current capabilities.

\section{EXPERIMENTAL RESULTS-SCREENING NONLINEARITY}

In this section we compare our results of the theoretical analysis with the experimental results presented in Ref. 25. However, some modification of the theory needs to be made, since the experiment employed a photorefractive strontium barium niobate (SBN) crystal exhibiting the screening nonlinearity, ${ }^{34-36}$ which is different from the Kerr nonlinearity considered above. The explicit form of this nonlinearity is discussed in Appendix A. Also, the screening nonlinearity necessitates the use of a uniform, ordinarily polarized, background beam. The nonlinearity is affected by the intensity ratio between the nonlinearly propagating beam (extraordinary polarization) and this background beam. ${ }^{34-36}$ Therefore, in the experiment, the background beam is also recycled in the cavity to keep the nonlinearity strength independent of the feedback. In the numerical simulation we use Eq. (A2) of Appendix A for $\Delta n$, calculating the integral numerically at each step. For the analytical part, the approximate expression (A3) is used. Performing the linearization of the nonlinear Schrödinger equations in the same manner as in Appendix A, while taking into account the boundary conditions and the fact that the total background intensity equals the intensity of the incoming background beam $I_{b}$ multiplied by a factor $1 /\left(1-\varepsilon^{2}\right)$, results in a set of equations describing the evolution of the perturbations:

$$
i \frac{\partial \bar{\phi}_{i}}{\partial \zeta}+\frac{\partial^{2} \bar{\phi}_{i}}{\partial \xi^{2}}+\left(1-\varepsilon^{2}\right) \frac{\psi_{0}^{2}}{1+\psi_{0}^{2}} \sum_{j} \varepsilon^{2 j}\left(\bar{\phi}_{j}+\bar{\phi}_{j}^{*}\right)=0,
$$

where $\psi_{0}^{2}$ is the mean intensity of the incoming beam in units of $I_{b}$ and $\bar{\phi}_{i}$ is defined as before. This set of equations is identical in form to Eq. (7), differing only in the pump parameter, which is here $\left(1-\varepsilon^{2}\right)\left[\psi_{0}^{2} /\left(1+\psi_{0}^{2}\right)\right]$ instead of $\psi_{0}^{2}$. This obviously changes only the exponential gain factor, which becomes

$$
g(q)=|q|\left(\frac{2 \psi_{0}^{2}}{1+\psi_{0}^{2}}-q^{2}\right)^{1 / 2} .
$$

This result is independent of the feedback in the cavity, as intended. The maximal gain factor $g_{\max }=\psi_{0}^{2} /\left(1+\psi_{0}^{2}\right)$ occurs for the spatial frequency $q_{\max }=\left[\psi_{0}^{2} /\left(1+\psi_{0}^{2}\right)\right]^{1 / 2}$, and hence this spatial frequency will dominate the MI process in the cavity. Substituting this into the expres- 


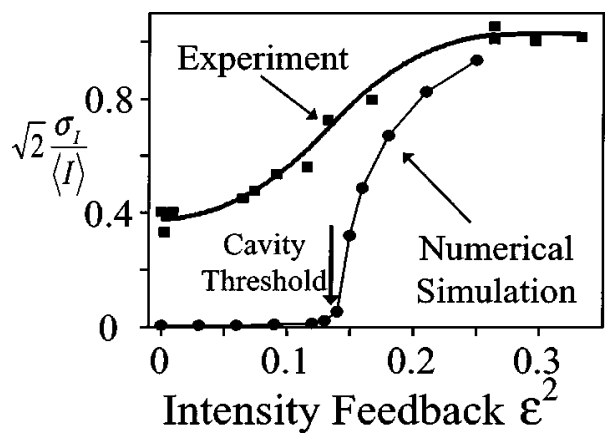

Fig. 7. Comparisons between the modulation depth calculated for the numerical simulation (circles) and experimental results (squares) with the screening nonlinearity. The cavity threshold is marked by an arrow.
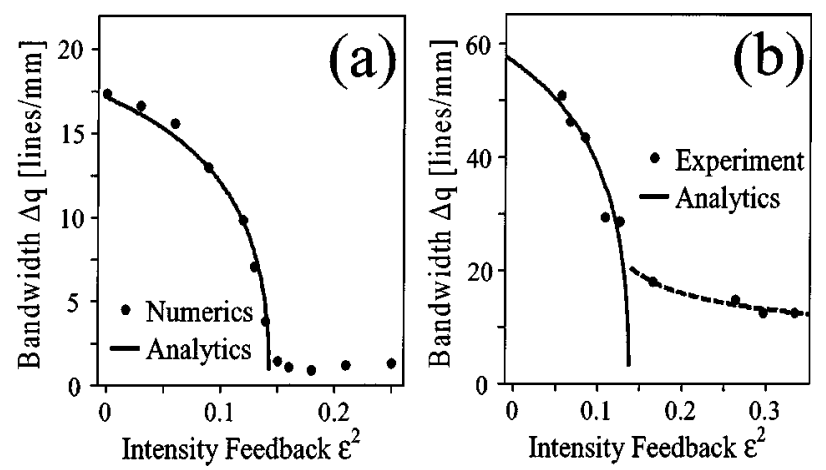

Fig. 8. Bandwidth of the perturbations' spatial spectrum versus intensity feedback in the (a) numerical simulation and (b) experimental results taken from Ref. 25, marked by circles. The solid curves in both figures show the theoretical dependence of the bandwidth based on the analytic solution. The dashed curve above the threshold in (b) is a guide to the eye.

sion for the spectral density of the perturbation [expression (14)] gives the feedback threshold value of

$$
\varepsilon_{\mathrm{th}}^{2}=\exp \left(-\frac{\psi_{0}^{2}}{1+\psi_{0}^{2}} L\right)
$$

We perform the numerical simulations under the same parameters used in the experiment: A 488-nm laser beam, with intensity ratio (signal/background) of 2.1, was sent into the cavity, and the nonlinear element was a $5.5-\mathrm{mm}\left(2.87\right.$ in dimensionless units $\left.{ }^{30}\right)$-long photorefractive $\mathrm{SBN}(60)$ crystal, with refractive index $n=2.36$ and electro-optic coefficient $180 \mathrm{pm} / \mathrm{V}$, biased by an external electric field of $340 \mathrm{~V} / \mathrm{cm}$. Substituting these parameters into the expression for the feedback threshold based on the analytic calculation above yields $\varepsilon_{\text {th }}{ }^{2}=14.3 \%$. Figure 7 shows the calculated visibility for the numerical simulations (marked by circles) compared with experimental results (squares) as a function of the intensity feedback in the cavity. There are no fitting parameters. For both cases, the transition occurs at an intensity feedback of $\sim 14 \%$, as predicted by our linear stability analysis, and the trend of the curves is similar. However, because the initial noise in the experiment was much stronger than in the simulation, the visibility even without any feedback is higher, and the transition is smoother. As mentioned above, beyond the threshold, the visibility of the pattern should not depend on the initial noise, and, indeed, in that region the numerical simulation gives visibility values similar to the experimental results. Generally, the numerical simulation here gives similar behavior to that with the Kerr nonlinearity.

Next, we measure the spatial bandwidth of the perturbations as the feedback is increased, which is marked by circles in Fig. 8(a). We also compare the results with the analytical calculation (solid curve) and find again that our model describes well the cavity below threshold. Figure 8(b) compares the analytic calculation of the bandwidth with the experimental results presented in Ref. 25, both of which show similar line narrowing below the threshold. Again, the initial noise in the experiments is high; hence the initial width of the power spectrum is larger in the experiment [Fig. 8(b)] than in the numerical calculations [Fig. 8(a)]. Therefore, when coming to compare the analytic curves with the numerics and with the experiments, we multiplied each of these analytic curves by a different constant factor, to match the numerical and analytical values [Fig. 8(a)] and the experimental and analytical values [Fig. 8(b)] for the spatial bandwidth of the pattern at zero feedback. With this single fitting parameter, the analytic curves match nicely the numerical [Fig. 8(a)] and experimental [Fig. 8(b)] curves, coinciding for all values of bandwidth as a function of feedback, including the feedback threshold value. Above the threshold, the experiment and the numerical simulation exhibit the same behavior, showing further line narrowing as the feedback is increased. However, at high values of the feedback, the numerical results show some broadening of the spatial spectrum, as in the Kerr case (Fig. 6). As another comparison, the dominant spatial frequency predicted by our analytic calculation is $q_{\max }=[2.1 /(1+2.1)]^{1 / 2}=0.82$, which is 23 lines $/ \mathrm{mm}$ when transformed back into real dimensions. ${ }^{30}$ This value was verified by both the numerical simulations $(23 \pm 1 \mathrm{lines} / \mathrm{mm})$ and the experimental results $(26 \pm 3$ lines $/ \mathrm{mm})$ and was found to be within the error limits.

\section{CONCLUSION}

We have presented a theoretical study of modulation instability and pattern formation in a nonlinear cavity with temporally incoherent feedback. We have demonstrated the features of this cavity, such as a threshold above which the instability changes its nature and a highvisibility pattern forms. Similar to the temporal behavior of laser resonators, line narrowing of the spatial (transverse) spectrum occurs as the feedback is increased. Indeed, the behavior near the threshold has much in common with second-order phase transitions. However, the incoherent cavity has no resonant frequencies, so there is no frequency-detuning effect near transition, as in the coherent case. We have analyzed the dynamics of the system below threshold, both numerically and by using linear perturbation theory, for both Kerr and saturable nonlinearities. Above threshold, we found that the modulation amplitude depends only on feedback and is independent of the initial noise level in the system. Both analytics and numerics give the same cavity threshold and line-narrowing behavior, which agree (within error 
limits) with the experimental observations reported in Ref. 25. Which one of the physical assumptions breaks down above the cavity threshold is still an open question, and an analytical description of this cavity above the threshold as well as other properties of this phase transition, is yet to be sought for. Also, it is interesting to examine cases of intermediate coherence length, when there is coherent interaction between some finite number of cycles. Finally, the direct continuation of this paper is the investigation of a spatially and temporally incoherent cavity as was first analytically studied (below the threshold) in Ref. 26 and also later experimentally. ${ }^{27}$

\section{APPENDIX A}

In this appendix we rederive the form of the nonlinearity involved in the MI process in a uniformly illuminated, electrically biased, photorefractive crystal, as used in the experiment of Ref. 25.

Consider a biased photorefractive crystal illuminated by an intensity distribution $\bar{I}(x)$ of an extraordinarily polarized electromagnetic field and a uniform (ordinarily polarized) background intensity $I_{b}$. To the lowest order and for low intensities, the total dc electric field inside the material is given by $^{34,35}$

$$
E(x)=-\frac{V}{l} \frac{\eta}{[1+I(x)]},
$$

where $I$ is the intensity distribution in units of $I_{b}, l$ is the transverse length of the crystal, $V$ is the applied voltage, and

$$
\eta \equiv\left(\frac{1}{l} \int_{0}^{l} \frac{\mathrm{d} x}{1+I}\right)^{-1}
$$

Through the electro-optic effect, this modulated electric field induces a spatially modulated change in the refractive index, given by

$$
\Delta n(x)=-\Delta n_{0}\left(\frac{1}{l} \int_{0}^{l} \frac{\mathrm{d} x}{1+I}\right)^{-1} \frac{1}{(1+I)},
$$

with $\Delta n_{0} \equiv \frac{1}{2} n_{b}{ }^{3} r_{\text {eff }}(V / l)$, where $n_{b}$ is the background refractive index and $r_{\text {eff }}$ is the effective electro-optic coefficient. Notice that this form is applicable only to a onedimensional intensity distribution and neglects higherorder effects, such as diffusion fields. ${ }^{36}$

In order to understand the nature of this nonlinearity and its contribution to the MI process, let us assume an almost uniform intensity distribution with small oscillations around a mean intensity $I_{0}$. In this case, the term in the integral of Eq. (A2) can be approximated as constant, and the nonlinear change in the refractive index takes a simpler form:

$$
\Delta n(x)=-\Delta n_{0} \frac{1+I_{0}}{1+I(x)} .
$$

We now examine the onset of the instability by substituting $\Delta n(x)$ into the dimensionless nonlinear wave equation:

$$
i \frac{\mathrm{d} \Psi}{\mathrm{d} \zeta}+\frac{\mathrm{d}^{2} \Psi}{\mathrm{d} \xi^{2}}-\frac{1+I_{0}}{1+|\Psi|^{2}} \Psi=0,
$$

where $\zeta$ and $\xi$ are the propagation and transverse coordinates, respectively, ${ }^{30}$ and $\Psi(\xi, \zeta)$ is the slowly varying envelope of the electromagnetic field inside the material, with $|\Psi|^{2}$ as the light intensity. We linearize Eq. (A4) and find the dynamics of the small perturbations in the usual way: We first choose the ansatz $\Psi=\left[\psi_{0}\right.$ $\left.+\psi_{1}(\xi, \zeta)\right] \exp (i \Gamma \zeta)$, where $\psi_{0}$ is the square root of the mean intensity $I_{0}$ and chosen, without the loss of generality, to be real; $\psi_{1}$ describes the propagating perturbation; and $\Gamma$ is their mutual propagation constant. Substituting this into Eq. (A4), expanding up to first order in $\psi_{1}$, and collecting terms of the same order separately, we find the following:

1. $\Gamma=-1$, reflecting the fact that in a uniformly illuminated photorefractive material the space-charge field is not modulated. Therefore the internal field is just the applied field $(V / l)$, and the change in the wave number (propagation constant) comes through the electro-optic effect alone [in other words, $\Delta n(x)=-\Delta n_{0}$, to first order].

2 . The evolution of the propagating perturbation inside the photorefractive medium is governed by

$$
i \frac{\mathrm{d} \psi_{1}}{\mathrm{~d} \zeta}+\frac{\mathrm{d}^{2} \psi_{1}}{\mathrm{~d} \xi^{2}}+\frac{\psi_{0}^{2}}{1+\psi_{0}^{2}}\left(\psi_{1}+\psi_{1}^{*}\right)=0
$$

This linearized equation describes the propagation of $\psi_{1}$ with a source term, pumped by $\psi_{0}$, counteracting the diffraction term and enhancing the perturbation along propagation.

Comparing this result with Kerr nonlinearity, we find that the pump strength, given in the Kerr medium by $\psi_{0}^{2}$, is replaced here by $\psi_{0}^{2} /\left(1+\psi_{0}^{2}\right)$. This form is of saturable nature, its maximum value being 1 , meaning that the growth rate of the perturbations will saturate in high signal-to-background ratios. However, the nonlinearity itself does not saturate, resembling in nature to that occurring with dark photorefractive solitons ${ }^{34}$ (rather than that found in bright photorefractive solitons). This results from the fact that increasing $I_{0}$ enhances the electric photocurrent going through the crystal. The outcome of the linear stability analysis presented here suggests that the MI process under the photorefractive screening nonlinearity has an upper bound to its strength. However, the nonlinear gain is monotonically increasing with intensity ratio, and hence working at high intensity ratios does not suppress MI, as was previously suggested (e.g., Ref. 31).

\section{ACKNOWLEDGMENTS}

This research was partially supported by the GermanIsraeli Deutsche-Israelische-Projektkooperation program, by the Israeli Science Foundation and by the U.S.Israel Binational Science Foundation. 


\section{REFERENCES AND NOTES}

1. For an extensive review on pattern formation in various nonlinear systems, see M. C. Cross and P. C. Hohenberg, "Pattern formation outside of equilibrium," Rev. Mod. Phys. 65, 851-1112 (1993).

2. A. Hasegawa, "Generation of a train of soliton pulses by induced modulational instability in optical fibers," Opt. Lett. 9, 288-290 (1984).

3. F. T. Arecchi, S. Boccaletti, and P. L. Ramazza, "Pattern formation and competition in nonlinear optics," Phys. Rep. 318, 1-83 (1999).

4. P. Mandel and M. Tlidi, "Transverse dynamics in cavity nonlinear optics (2000-2003)," J. Opt. B 6, R60-R75 (2004).

5. C. Tamm, "Frequency locking of two transverse optical modes of a laser," Phys. Rev. A 38, 5960-5963 (1988).

6. K. Staliunas, G. Slekys, and C. O. Weiss, "Nonlinear pattern formation in active optical systems-shocks, domains of tilted waves, and cross-roll patterns," Phys. Rev. Lett. 79, 2658-2661 (1997).

7. G. L. Oppo, M. Brambilla, and L. A. Lugiato, "Formation and evolution of roll patterns in optical parametric oscillators," Phys. Rev. A 49, 2028-2032 (1994).

8. M. Vaupel, A. Maitre, and C. Fabre, "Observation of pattern formation in optical parametric oscillators," Phys. Rev. Lett. 83, 5278-5281 (1999).

9. J. Scheuer and M. Orenstein, "Optical vortices crystals: spontaneous generation in nonlinear semiconductor microcavities," Science 285, 230-233 (1999).

10. S. R. Liu and G. Indebetouw, "Periodic and chaotic spatiotemporal states in a phase-conjugate resonator using a photorefractive $\mathrm{BaTiO}_{3}$ phase-conjugate mirror," J. Opt. Soc. Am. B 9, 1507-1520 (1992).

11. K. Staliunas, M. F. H. Tarroja, G. Slekys, and C. O. Weiss, "Analogy between photorefractive oscillators and class-A lasers," Phys. Rev. A 51, 4140-4151 (1995).

12. W. J. Firth and A. J. Scroggie, "Optical bullet holes-robust controllable localized states of a nonlinear cavity," Phys. Rev. Lett. 76, 1623-1626 (1996).

13. K. Ikeda, H. Daido, and O. Akimoto, "Optical turbulencechaotic behavior of transmitted light from a ring cavity," Phys. Rev. Lett. 45, 709-712 (1980).

14. M. Haelterman, S. Wabnitz, and S. Trillo, "Additivemodulation-instability ring laser in the normal dispersion regime of a fiber," Opt. Lett. 17, 745-747 (1992).

15. S. Coen and M. Haelterman, "Modulational instability induced by cavity boundary conditions in a normally dispersive optical fiber," Phys. Rev. Lett. 79, 4139-4142 (1997).

16. S. J. Bentley, R. W. Boyd, W. E. Butler, and A. C. Melissinos, "Spatial patterns induced in a laser beam by thermal nonlinearities," Opt. Lett. 26, 1084-1086 (2001).

17. S. J. Jensen, M. Schwab, and C. Denz, "Manipulation, stabilization, and control of pattern formation using Fourier space filtering," Phys. Rev. Lett. 81, 1614-1617 (1998).

18. M. D. Iturbe-Castillo, M. Torres-Cisneros, J. J. SanchezMondragon, S. Chavez-Cerda, S. I. Stepanov, V. A. Vysloukh, and G. E. Torres-Cisneros, "Experimental evidence of modulation instability in a photorefractive $\mathrm{Bi}_{12} \mathrm{TiO}_{20}$ crystal," Opt. Lett. 20, 1853-1855 (1995).

19. A. V. Mamaev, M. Saffman, D. Z. Anderson, and A. A. Zozulya, "Propagation of light beams in anisotropic nonlinearmedia: from symmetry breaking to spatial turbulence," Phys. Rev. A 54, 870-879 (1996).

20. M. I. Carvalho, S. R. Singh, and D. N. Christodoulides,
"Modulational instability of quasi-plane-wave optical beams biased in photorefractive crystals," Opt. Commun. 126, 167-174 (1996).

21. W. J. Firth and C. Paré, "Transverse modulational instabilities for counterpropagating beams in Kerr media," Opt. Lett. 13, 1096-1098 (1988).

22. T. Honda, "Hexagonal pattern formation due to counterpropagation in $\mathrm{KNbO}_{3}$," Opt. Lett. 18, 598-600 (1993).

23. L. A. Lugiato and C. Oldano, "Stationary spatial patterns in passive optical systems: two-level atoms," Phys. Rev. A 37, 3896-3908 (1988).

24. L. A. Lugiato and R. Lefever, "Spatial dissipative structures in passive systems," Phys. Rev. Lett. 58, 2209-2211 (1987).

25. T. Carmon, M. Soljacic, and M. Segev, "Pattern formation in a cavity longer than the coherence length of the light in it," Phys. Rev. Lett. 89, 183902 (2002).

26. H. Buljan, M. Soljacic, T. Carmon, and M. Segev, "Cavity pattern formation with incoherent light," Phys. Rev. E 68, 016616 (2003).

27. T. Carmon, H. Buljan, and M. Seger, "Spontaneous pattern formation in a cavity with incoherent light," Opt. Express 12, 3481-3487 (2004), http://www.opticsexpress.org.

28. A. Yariv, Quantum Electronics, 3rd ed. (Wiley, New York, 1988), p. 579.

29. This interaction resembles cross-phase modulation [See M. Haelterman, S. Trillo, and S. Wabnitz, "Polarization multistability and instability in a nonlinear dispersive ring cavity," J. Opt. Soc. Am. B 11, 446-456 (1994)], yet it leads to different phenomena. In the cross-phase modulation the two orthogonal polarizations interact through the sum of their intensities alone, but, in contrast to our case, the fields of each polarization are coherent. Therefore, in that case, each polarization has its own set of resonant frequencies, and the pattern formation process generally depends on these frequencies.

30. In transforming to the dimensionless equation, we use $x_{0}$ $=\lambda /\left[2 \pi\left(2 n \Delta n_{0}\right)^{1 / 2}\right]$ and $z_{0}=\lambda / 2 \pi \Delta n_{0}$ as the characteristic transverse and propagation scales, respectively, where $\lambda$ is the wavelength in vacuum, $n$ is the material refractive index, and $\Delta n_{0}$ is the typical scale of the nonlinear change in the refractive index.

31. M. Soljacic, M. Segev, T. Coskun, D. N. Christodoulides, and A. Vishwanath, "Modulation instability of incoherent beams in noninstantaneous nonlinear media," Phys. Rev. Lett. 84, 467-470 (2000).

32. We define the bandwidth $\Delta q$ as the standard deviation of the spatial power density: $\Delta q=\left[\int_{0}^{\infty}(q\right.$ - $\left.\langle q\rangle)^{2} S(q) \mathrm{d} q\right]^{1 / 2} / \int_{0}^{\infty} S(q) \mathrm{d} q$, where $S(q)$ is the perturbations' spatial power spectrum and $\langle q\rangle$ $=\int_{0}^{\infty} q S(q) \mathrm{d} q / \int_{0}^{\infty} S(q) \mathrm{d} q$ is the mean transverse wave number.

33. H. Haken, Synergetics: An Introduction, 3rd ed. (SpringerVerlag, Berlin, 1983), p. 229.

34. M. Segev, G. C. Valley, B. Crosignani, P. DiPorto, and A. Yariv, "Steady-state spatial screening solitons in photorefractive materials with external applied field," Phys. Rev. Lett. 73, 3211-3214 (1994).

35. M. Segev, M. Shih, and G. C. Valley, "Photorefractive screening solitons of high and low intensity," J. Opt. Soc. Am. B 13, 706-718 (1996).

36. D. N. Christodoulides and M. I. Carvalho, "Bright, dark, and gray spatial soliton states in photorefractive media," J. Opt. Soc. Am. B 12, 1628-1633 (1995). 